discovered by trial and error, but David Liu and his co-workers at Harvard University in Cambridge, Massachusetts, have devised a method for screening large numbers of reactant pairs in organic solvents to find out which join up under different conditions.

It involves tethering two different chemical groups to a single strand of DNA that is unique to each combination. If two groups react, the product remains stuck to the pairspecific DNA, even though the DNA's link to one of the groups is cut.

A chemical hook attached to one reactant group sticks to the surface of magnetic beads, which can then be separated. But the hooks only retain the DNA of pairs that have reacted - otherwise the earlier cleavage process cuts it free. DNA hybridization then reveals the identities of the product-forming pairs.

\section{PLANT EVOLUTION}

\section{Taking after mother}

Science 318, 1134-1136 (2007)

Mother plants can transmit information about their environment to their offspring, thereby increasing their progeny's fitness.

Laura Galloway at the University of Virginia in Charlottesville and Julie Etterson at the University of Minnesota-Duluth studied Campanulastrum americanum, a small forest plant that grows both in sunny spots and in the shade of the forest canopy. Its seeds tend to sprout near the mother plant, within the same patch of sun or shade.

When the researchers grew plants in the opposite light environment from that of their mothers, the population grew at a slower rate. This suggests that maternal plants cue their offspring about the light environment they are likely to experience. Because this signal can change each generation, it is a flexible way of enhancing the success of offspring.

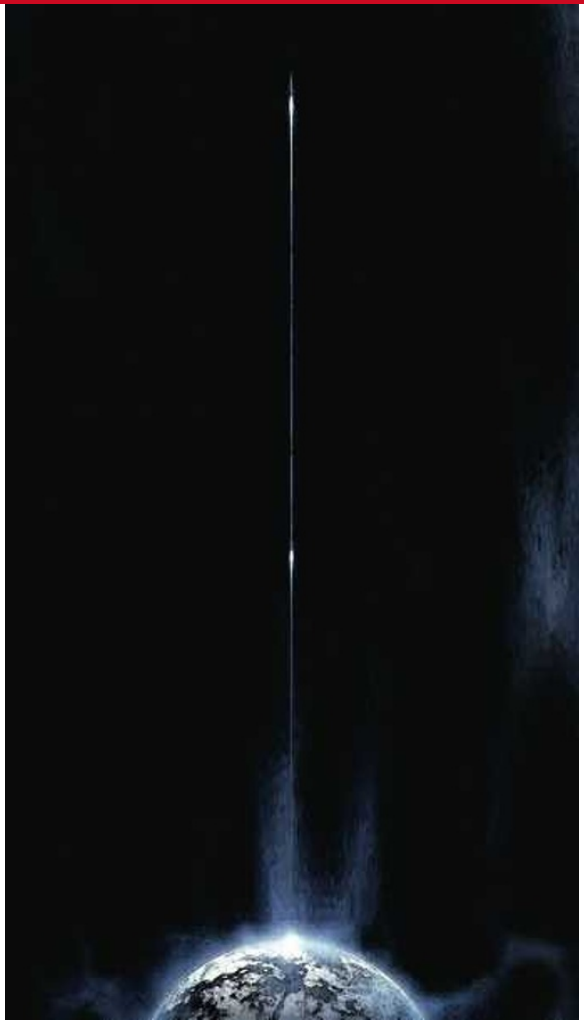

CELL BIOLOGY

\section{Size control}

J. Cell Biol. doi: 10.1083/jcb.200708054 (2007)

Fission yeast regulates the size of its nucleus to stay in sync with the volume of its cytoplasm, researchers have found.

How cells control organelle size is a century-old question. Frank Neumann and Paul Nurse at the Rockefeller University in New York measured nuclear and cellular volumes in the fission yeast Schizosaccharomyces pombe. Using various mutants, they found similar nucleus-to-cell volume ratios over a 35 -fold variation in cell size and a 16-fold difference in DNA content.

And from studies of cells with four nuclei, they suggest that the cytoplasmic environment that the nucleus resides in is an important regulator of its size. The molecular basis of this control remains unknown.

\section{MATERIALS SCIENCE}

\section{Out of reach}

Nanotoday 2, 44-47 (2007)

Space elevators (depicted left) are a mainstay of science fiction. But advances in nanotechnology have spurred Nicola Pugno at Torino Polytechnic in Italy to consider whether shooting a payload into space up a 100,000-kilometre-long cable may one day be possible.

Previously, he worked out that the tensile strength of a huge carbon nanotube bundle would drop from 100 gigapascals to 80 gigapascals, were each nanotube to have one atom out of place. Now his calculations show that each rod would inevitably contain at least one defect four atoms long, making it less than 45 gigapascals strong and weaker than the 63 gigapascals required for a line of uniform thickness. Manufacturing constraints would force engineers to make a cable up to 600 times fatter at the height of its geosynchronous orbit.

\section{QUANTUM PHYSICS}

\section{Early jiggles}

Phys. Rev. Let. 99, 201301 (2007)

A line of ions could provide clues about the early Universe. Ralf Schützhold at the Technical University of Dresden in Germany and his colleagues have calculated that quantum fluctuations in the vibrations of a column of trapped magnesium ions are mathematically similar to the period of rapid expansion that followed the Big Bang.

Stretching and squeezing the column should cause the quantum fluctuations to become real vibrations, in much the same way that the stretching of space-time created matter and energy in the young cosmos. The group is now trying an experiment to see how well the analogy holds up.

\section{JOURNAL CLUB}

\section{Arturo Zychlinsky \\ Max Planck Institute for Infection Biology, Berlin, Germany.}

A microbiologist wonders how antimicrobial peptides beat infection.

My group is interested in why, although people often pick up infections and sometimes become ill, they almost always recover. Recovery is the result of a fantastically efficient immune system that relies in part on proteins and peptides that kill microbes.

Antimicrobial peptides were discovered in systematic searches for potential drugs, and there are several types. Most are cationic and bind to the anionic surface of microbes. Recently, Roberto Lande at the University of Texas in Houston and his colleagues convincingly showed that one antimicrobial peptide, LL-37, can also bind DNA and serve as an activator for other immune cells (R. Lande et al. Nature 449, 564-569; 2007).

LL-37 is one of several antimicrobial peptides that do more than kill microbes and activate immune cells. Its other functions include chemoattraction and wound repair. But, as its name indicates, LL-37 has only 37 amino acids. It is plausible that its multiple effects on the host are due to its interaction with specific receptors that, in combination with other signals, result in diverse biological functions. More intriguing, however, is the fact that LL-37 kills microbes in the first place.

The question is whether LL-37 and other antimicrobial peptides truly function as bacterial killing agents in the host. Their antimicrobial activity has so far been demonstrated only in vitro, where it might be a reflection of their cationic character. Another more attractive possibility is that infections are such an important threat to the host that, during evolution, many cationic proteins with diverse function were coopted to serve as antimicrobial agents. If we do have many ways to kill microbes, maybe it is not so surprising that we often recover from infections.

Discuss this paper at http://blogs. nature.com/nature/journalclub 